International Journal of Research in Nursing 5 (1): 1-2, 2014

ISSN: 1949-0194

(C) 2014 V. Chaban, This open access article is distributed under a Creative Commons Attribution

(CC-BY) 3.0 license

doi:10.3844/ijrnsp.2014.1.2 Published Online 5 (1) 2014 (http://www.thescipub.com/ijrn.toc)

\title{
CHRONIC PELVIC PAIN: FOCUS ON ETIOLOGY AND MODULATION
}

\author{
Victor Chaban
}

Charles R. Drew University and University of California, Los Angeles, USA

Chronic Pelvic Pain (CPP) is defined as pelvic pain in the same location for at least 6 months, affects up to $25 \%$ of women at some time in their lives and can result in dysmenorrhea, dyspareunia, menstrual irregularities, back pain, gastrointestinal and genitourinary symptoms and reduced fecundity. Some of the most common causes of CPP are endometriosis, vulvodynia and abdominal wall and pelvic floor myalgia and neuropathy. Most women with complaints of pelvic pain will undergo laparoscopy to both diagnose and treat these diseases, but laparoscopy is often is unsuccessful due to lack of intraperitoneal pathology or altered pain processing. Pain out of proportion to identifiable pathology is the most immediate and dramatic consequence of disorders associated with CPP and is responsible for a highly negative impact on quality of life and substantial workforce loss. Due to the alarming situation and unmet need, many countries have launched a call for more focused research on improving the treatment of CPP syndrome.

Pain is subjectivefeeling and thus difficult to be standardized and parameterized for scientific analysis. Often the etiology of CPP is not clear, as there are many symptoms of the reproductive system, gastrointestinal and urinary tracts, musculoskeletal, neurological and psychological systems that often co-occur in the same patient. Pain is also the subjectiveexperience which can occur, particularly in its chronic manifestation, in the absence of physiological findings that explain its persistence or severity. Because of the inherent subjectivity of pain, there is a wide disparity among individuals in the way that they experience the pain generated by what seem to be similar stimuli. Pain is a complex and individualexperience that is often difficult for patients to fully describe using conventional pain intensity.

There are two essential components of pain: discriminative and affective. The discriminative component includes the ability to identify the stimulus as originating from somatic or visceral tissue, determine some of the physical properties of the stimulus and localize it in space, time and along a continuum of intensities. The affective component is the experience which motivates escape, avoidance and protective behavior. A consideration of pain mechanisms must also include the neural systems modulating pain, for it is well known that pain can be profoundly influenced by other somatic stimuli and by attentional, emotional and cognitive factors. The history and physical examination are crucial in evaluating a suffering patient and must address all of the possible systems potentially involved in CPP. Suffering patients may initially have only one pain source in the pelvis, but a multitude of mechanisms involving the peripheral and central nervous system can lead to the development of painful sensations from other adjacent organs such as lower colonic pain associated with IBS. There is often no clear relationship between the severity of the CPP and pathology in the pelvic viscera, including reproductive tract (ovaries and uterus), urinary bladder and colon. Patients with CPP who display more than one demonstrable pain generator are susceptible to development of the CPP syndrome, either through previous trauma or other genetic or environmental factors.

Most diagnoses associated with CPP have a high rate of recurrence and all are considered to be chronic conditions with a relapsing course. There is often no clear relationship between the severity of the CPP and pathology in the pelvic viscera. Vicero-somatic and viscero-viseralhyperalgesia and allodynia result in the spread of a perception of pain from an initial site to adjacent areas. CPP patients may initially have only one pain source in the pelvis, such as the uterus in dysmenorrhea or endometriosis implants, but a multitude of mechanisms involving the peripheral and central nervous system can lead to the development of painful 
sensations from other adjacent organs. Often the etiology of visceral pain is not clear, as there are many symptoms of the reproductive system, gastrointestinal and urinary tracts, musculoskeletal, neurological and psychological systems that often co-occur in the same patient. The variation of pain symptoms and pain perception and behavioral responses to pain in these patients is poorly understood. The treating clinician is often tempted to take a unidimensional approach and focus on one organ system and ignore the psychological and behavioral manifestations of the chronic pain. The incidence of persistent, episodic, or chronic visceral pain are more prevalent in females thus defining the site(s) and mechanisms through which female steroid hormones modulate visceral nociception is an important step in understanding the gender differences in pain perception and in designing appropriate therapies for females. One such mechanism may be the convergence of nociceptive stimuli and estrogen input on the primary afferent neurons which innervate viscera. Based on our recent results, it is likely that sex steroid receptors expressed in primary afferent neurons may modulate nociceptive signaling (Chaban, 2012a; 2012b; 2013).

CPP patients have significantly more depression, psychological and somatic complains and more often give a history of physical, sexual or emotional abuse or trauma. CPP adversely affects mood, social and professional life and general well-being. These quality of life issues can affect severity of pain, degree of impairment resulting from a painful condition and success of pain treatment modalities in alleviating pain. The treating clinician is often tempted to take a unidimensional approach and focus on one organ system and ignore the psychological and behavioral manifestations of the chronic pain. CPP syndrome implies the pain symptoms are not just a manifestation of a peripheral pelvic abnormality, but a complex entity in which traditional therapy is less effective due to altered central nervous system processing of pain signals.

\section{REFERENCES}

Chaban, V., 2012a. Primary afferent nociceptors and visceral pain, endometriosis-basic concepts and current research trends. InTech, pp: 367-381.

Chaban, V., 2012b. Role of primary afferent nociceptors in visceral pain associated with Irritable Bowel Syndrome. In: "Irritable Bowel Syndrome: Symptoms, Diagnosis and Treatment Options", NOVA Publishers.

Chaban, V., 2013. Estrogen modulation of visceral nociception. Current Trends Neurology, 2013: 51-55. 